\title{
A Patient with Bilateral Sciatic Neuropathies
}

\author{
Erin K. O’Ferrall, Kevin Busche, Peter Dickhoff, Rana Zabad, Cory Toth
}

Can. J. Neurol. Sci. 2007; 34: 365-367

A 29-year-old female was found in her home in a seated position in a floor-level cupboard with a depressed level of consciousness after ingesting an overdose of amitriptyline and alcohol the night before. She was transferred to hospital and treated for tricyclic antidepressant overdose and was found to have rhabdomyolysis. Her creatine kinase peaked at 70000 units/L.

Within 24 hours after admission, the patient recovered her normal level of consciousness and complained of bilateral hip, groin and buttock pain exacerbated by movement. She also had weakness, paresthesias and numbness in both lower extremities, more pronounced on the right. Following initial consideration of other possible causes, Neurology was consulted.

Her examination demonstrated normal cranial nerves and upper extremity function. The patient had pain with passive flexion of the hips. There was bilateral weakness of leg extension $(2 / 5)$, hip abduction (3/5), knee flexion (3/5), ankle dorsiflexion (4-/5), plantar flexion (4-/5), foot inversion (4-/5), foot eversion (4-/5), toe dorsiflexion (4-/5), and toe plantar flexion (4-/5), with each slightly worse for the right leg. Deep tendon reflexes were absent at both ankles but present and symmetric elsewhere, including at the knees and adductor muscles. Plantar responses were flexor. Sensory exam revealed decreased pinprick sensation over the right foot and the lateral lower right leg, along with minimally decreased pin prick sensation over the left sole and medial aspect of the left foot.

On day seven of admission, an enhanced Computed Tomography (CT) scan of the pelvis revealed moderately large areas of low attenuation in the gluteus muscles bilaterally, right greater than left (see Figure). The normal fat planes surrounding the sciatic nerve in the sciatic notch (figure, arrows) are obscured by low attenuation fluid or hematoma. The low attenuation is rounded and more prominent on the left than on the right. The low attenuation fluid or hematoma may reflect tracking from the gluteal muscle or direct nerve injury. The findings were consistent with areas of muscle injury, infarct or hematoma due to pressure necrosis and reflected the degree of pressure injury to the gluteal and buttock area. No compartment syndrome was felt to be present by either ICU specialists or surgical consultants. Surgical decompression was considered but not performed. An ultrasound of the lower extremities was negative for deep venous thrombosis. The patient was discharged 11 days after admission, using a wheelchair to mobilize.

The patient returned for nerve conduction studies nine weeks after her initial presentation. At that time she was taking gabapentin and oxycodone for neuropathic pain over both distal legs. She required a cane to ambulate and wore a right ankle

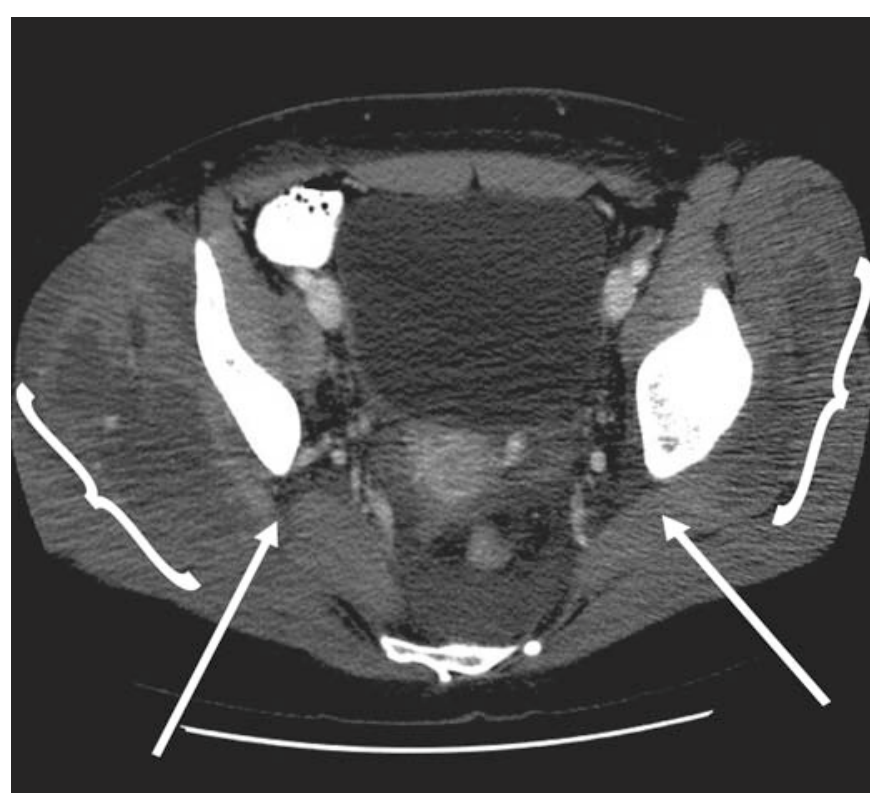

Figure: Two selected axial computed tomography images of the pelvis. Moderate size areas of low attenuation in the gluteal muscles (braces) and relation to sciatic notch (arrows).

brace for foot drop. Her physical exam demonstrated loss of muscle bulk at the right tibialis anterior, right gastrocnemius and the right extensor digitorum brevis. Muscle power was normal in the left lower extremity but there was persistent weakness in the right lower extremity for muscles innervated by the sciatic nerve. Hip extension was weak, but hip flexion and adduction were of normal power.

From the Department of Clinical Neurosciences (EKO, KB, RZ, CT), University of Calgary and the Calgary Health Region; Department of Radiology (PD) and Calgary Health Region, Calgary, Alberta, Canada.

RECEIVED SEPTEMBER 26, 2006. ACCEPTED IN FINAL FORM APRIL 16, 2007. Reprint requests to: Cory Toth, HMRB Room 155, University of Calgary, Dept. of Clinical Neurosciences, 3330 Hospital Drive NW, Calgary, Alberta, T2N 4N1, Canada. 
Sensory and motor nerve conduction studies were performed in the upper and lower extremities (see Table). The superficial peroneal and sural sensory nerve action potential (SNAP) responses were absent bilaterally. The tibial and peroneal compound motor action potentials (CMAPs) were absent on the right. The left peroneal CMAP was small while its distal latency was in the upper range of normal. For the right peroneal motor nerve, stimulation below the knee with recording electrodes placed over the tibialis anterior yielded a very small CMAP of $0.1 \mathrm{mV}$ with a prolonged latency of $9.9 \mathrm{~ms}$. Importantly, femoral CMAP and saphenous nerve SNAP responses were normal.

EMG studies were attempted, but not completed due to patient intolerance.

The patient was diagnosed with bilateral sciatic neuropathies, more severe on the right.

\section{Discussion}

Unilateral sciatic neuropathy has been well described as a result of injuries to the sciatic nerve in the gluteal region. ${ }^{1}$ Causes have included trauma, a perioperative complication of hip or knee surgery, surgery while in the lithotomy or sitting position, a posterior compartment syndrome of the thigh, compression due to local masses, subcutaneous injections and possibly due to the controversial piriformis syndrome. Bilateral sciatic neuropathy is more rare, ${ }^{2-9}$ but has been described with prolonged immobilization due to intoxication, ${ }^{2-7}$ while seated on the commode, while seated with legs hanging over a footboard, with prolonged yoga positions, and due to surgeries performed in the seated position. ${ }^{3-8}$ Imaging of the gluteal region has identified multifocal areas of enhancement in the vastus lateralis, adductor magnus \& gluteus maximus muscles in one other patient with sciatic neuropathy. ${ }^{7}$

Several mechanisms of sciatic nerve damage have been described: 1) stretching of the sciatic nerve and compression of the nerve against the sciatic notch; 2) compartment syndrome of the posterior thigh resulting in diffuse compression of the sciatic nerve; 3 ) local myonecrosis in the gluteal region with hematoma formation and direct focal compression of the sciatic nerve.

Although the exact mechanism of injury to our patient's sciatic nerves cannot be confirmed, we speculate that the nerve injury resulted from compression of the sciatic nerves by the low attenuation masses identified with CT scan. The non-specific appearance of the low attenuation masses likely represented areas of muscle necrosis or gluteal hematomas. Other mechanisms of sciatic nerve damage, such as ischemia (secondary to compression) remain possible.

Computed tomography, or even MRI, imaging of the gluteal region may assist in the diagnosis of bilateral sciatic nerve neuropathies in cases of prolonged immobilization such as in our case. Imaging of peripheral nerves can help elucidate the location, pattern (diffuse or focal) and extent of the lesion.

Table: Nerve conduction studies of the upper and lower extremities nine weeks after onset of symptoms

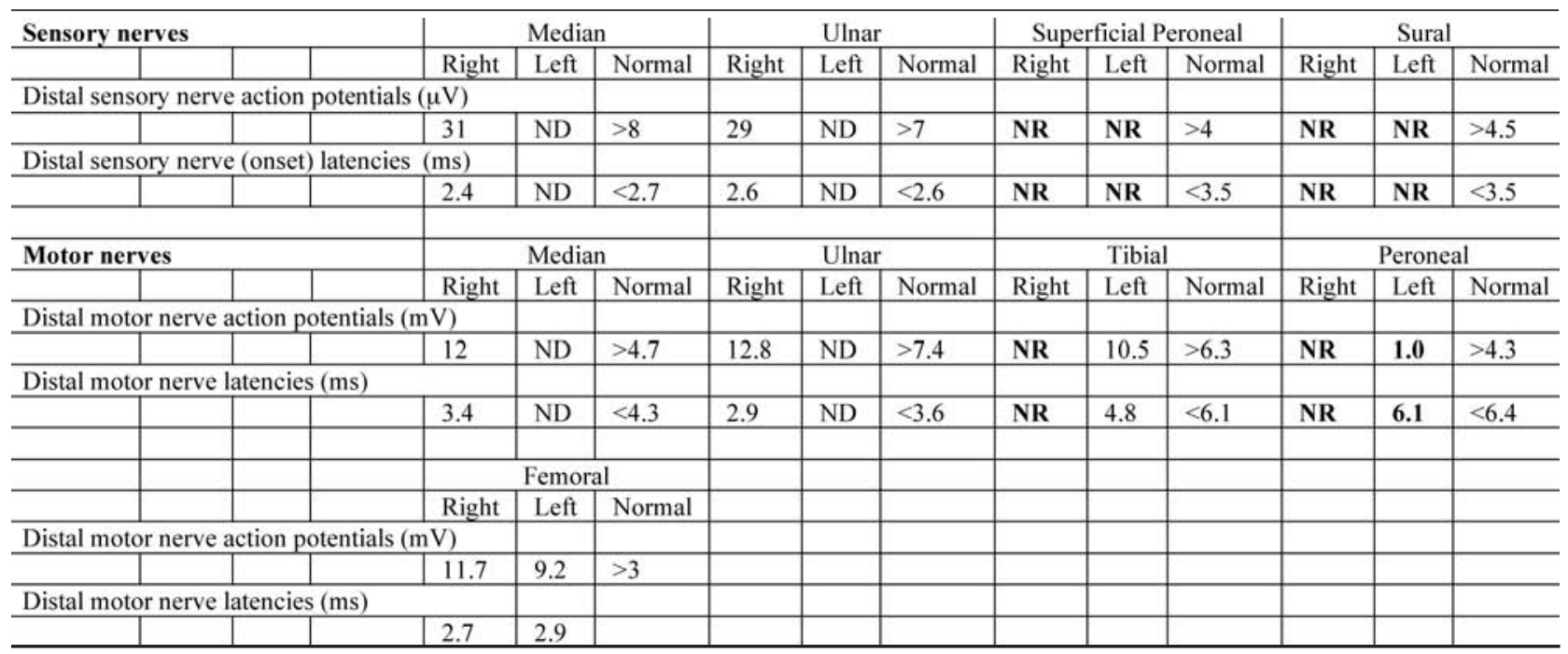

Stimulation and recording locations for each sensory nerve are median nerve, wrist to digit 2; ulnar nerve, wrist to digit 5; superficial peroneal nerve, lateral lower leg to ankle; sural nerve, lateral lower leg to heel. Stimulation and recording locations for each motor nerve are median, wrist to abductor pollicus brevis; ulnar nerve, wrist to adductor digiti minimi; tibial nerve, ankle to adductor hallicus brevis; peroneal nerve, ankle to extensor digitorum brevis; femoral nerve, inguinal area to anterior thigh. There was no significant drop in amplitude of the motor nerves with more proximal stimulation sites. Temperature was maintained at $32{ }^{\circ} \mathrm{C}$ or greater in the arms and $30.5{ }^{\circ} \mathrm{C}$ or greater in the legs for all testing. $\mathrm{NR}=$ no response. $\mathrm{ND}=$ not done. 
Peripheral nerve injuries can present with changes in size and signal of the nerve. Imaging can also help define changes in surrounding tissues leading to nerve compression, as identified in our case. An additional use of imaging is the identification of subacute or chronically denervated muscles with altered signal characteristics, atrophy and fatty infiltration. In our case, areas of low attenuation within the gluteal muscles likely represented ischemia and necrosis.

The optimal management of compressive sciatic neuropathies remains controversial and depends upon the underlying cause of compression. If a compartment syndrome is present, then fasciotomies and decompression are clearly indicated. ${ }^{10-12}$ Surgical exploration with decompression is recommended when mechanical or structural causes of sciatic nerve compression are suspected. This may occur as a late post-operative complication of hip arthroplasty due to migration of surgical hardware ${ }^{13}$ or due to entrapment by inflamed piriformis muscle ${ }^{14}$ or ossification. ${ }^{15}$ Good recovery after hematoma evacuation has been reported. ${ }^{16}$ Medical management has been suggested for patients with partial deficits or for those who are improving in terms of function and pain control. ${ }^{17}$

\section{REFERENCES}

1. Stewart JD. Focal peripheral neuropathies 3rd ed. Philidelphia: Lippincott Williams \& Wilkins: 2000

2. Scherer K, Skeen MB, Strine SA, Jeul VC. Hanging leg syndrome: combined bilateral femoral and sciatic neuropathies. Neurology. 2006; 66 (7): 1124-5.

3. Stewart JD, Angus E, Gendron D. Sciatic Neuropathies. BMJ. 1983; 287: 1108-9.

4. Tyrrell PJ, Feher MD, Rossor MN. Sciatic nerve damage due to toilet seat entrapment: another Saturday night palsy. J Neurol Neurosurg Psychiatry. 1988; 52:1113-15.

5. Walker M, Meekins G, Hu S-C. Yoga neuropathy: a snoozer. Neurologist. 2005; 11(3): 176-8.
6. Kornetzky L, Linden D, Berlit P. Bilateral sciatic nerve 'Saturday night palsy'. J Neurol. 2001; 248: 5.

7. Seet RCS, Lim ECH. Intravenous use of buprenorphine tablets associated with rhabdomyolysis and compressive sciatic neuropathy. Ann Emerg Med. 2006; 47(4): 396-7.

8. Plewnia C, Wallace C, Zochodne DW. Traumatic sciatic neuropathy: a novel cause, local experience, and a review of the literature. Trauma. 1999; 47(5): 986.

9. Sener M, Sener U, Yildiz M, Baki C. Bilateral traumatic hip dislocation with bilateral sciatic nerve injury. Arch Orthop Trauma Surg. 1997; 116: 225-6.

10. Vikram D, Thambiah J, Kada FHY, Prem Kumar V. Bilateral gluteal compartment syndrome. J Bone Joint Surg. 2005; 87: 2541-5.

11. Rodriguez Uranga JJ, Ucles Sanchez AJ, Perez Diaz JM. [Neuropathy of common sciatic nerve secondary to compartment syndrome as a complication after bariatric surgery] Neurologia. 2005; 20(2): 94-7.

12. Holbein O, Strecker W, Rath SA, Kinzl L. Compartment syndrome of the thigh with sciatic nerve paralysis. Unfallchirurg. 2000; 103(4): 275-80.

13. Steihl JB, Stewart WA. Late sciatic nerve entrapment following pelvic plate reconstruction in total hip arthroplasty. J Arthroplasty. 1998; 13(5): 586-8.

14. Uchio Y, Nishikawa U, Ochi M, Shu N, Takata K. Bilateral piriformis syndrome after total hip arthroplasty. Arch Orthop Trauma Surg. 1998; 117: 177-9.

15. Abayev B, Ha E, Cruise C. Sciatic nerve lesion secondary to compression by a heterotopic ossification in the hip and thigh region- an electrodiagnostic approach. Neurologist. 2005 May; 11(3): 184-6.

16. Weil Y, Mattan Y, Goldman V, Liebergall M. Sciatic nerve palsy due to hematoma after thrombolysis therapy for acute pulmonary embolism after total hip arthroplasty. J Arthroplasty. 2005 Apr; 21(3): 456-9.

17. Kline DG, Kim D, Midha R, Harsh C, Tiel R. Management and results of sciatic nerve injuries: a 24 year experience. J Neurosurg. 1998; 89(1): 13-23. 